hep-ph/9910408

\title{
Non-Universal Gaugino Phases and the LSP Relic Density
}

\author{
Shaaban Khalil \\ Departmento de Fisica Teórica, C.XI, Universidad Autónoma de Madrid, \\ 28049 Cantoblanco, Madrid, Spain. \\ and \\ Ain Shams University, Faculty of Science, Cairo 11566, Egypt.
}

\begin{abstract}
The cosmological relic density of the lightest supersymmetric particle (LSP) of type I string derived model is calculated. This model can accommodate large values of $\mathrm{CP}$ violating phases, and the electron and neutron electric dipole moments satisfy the experimental constraint. We show that the constraint from the electric dipole moment on the ratio between the gaugino masses implies that the mass of the LSP, which is bino like, is close to the lightest chargino. The co-annihilation between them is very important to reduce the LSP relic density to an interesting region. We show that the SUSY phases, although they are large, have no significant effect on the relic density and on the the upper bound imposed on the LSP mass. However, they are very significant for the detection rates. We emphasize that the phase of the trilinear coupling increase the direct and indirect detection rates.
\end{abstract}




\section{Introduction}

It is well known that supersymmetric (SUSY) models allow for new possibilities for CP violation. The soft SUSY breaking terms are in general complex. For instance, in the minimal supersymmetric model (MSSM), there are complex phases in the parameters $A$, $B$ (which are the coefficients of the SUSY breaking of the trilinear and bilinear couplings respectively), $M$ (the gaugino masses), and $\mu$ (the mass coefficient of the bilinear terms involving the two Higgs doublets). However, only two of these phases are physical (they can not be rotated away by all possible field redefinitions). These phases give large oneloop contributions to the electric dipole moments (EDM) of the neutron and electron which exceed the current limits

$$
\begin{aligned}
& d_{n}<6.3 \times 10^{-26} \mathrm{ecm}, \\
& d_{e}<4.3 \times 10^{-27} \mathrm{ecm} .
\end{aligned}
$$

Hence SUSY phases, which are generally quite constrained by (回), have to be of order $10^{-3}$ for SUSY particle masses of order the weak scale [1].

However it was pointed out that there are internal cancellations among various contribution to the EDM (including the chromoelectric and purely gluonic operator contributions) whereby allowing for large phases [2]. We have shown that in the effective supergravity (derived from string theory) such cancellations are accidental and it only occurs at few points in the parameter space [3]. Recently, it was argued that the nonuniversal gaugino masses and their relative phases are crucial for having sufficient cancellations among the contributions to EDMs 米. These cancellations have been studied in the framework of a D-brane model where $S U(3)_{C} \times U(1)$ and $S U(2)$ arise from one five

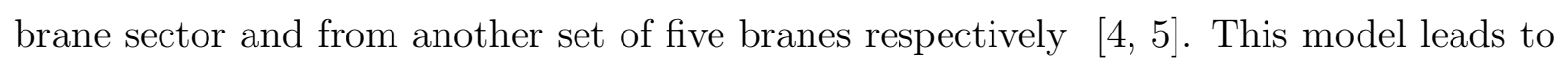
non universal gaugino mass which is necessary to ensure these cancellations.

In such a case one expects that these large phases have important impact on the lightest supersymmetric particle (LSP) relic density and its detection rates. In Ref. [6, [] the effect of SUSY phases on the LSP mass, purity, relic density, elastic cross section, and detection rates has been considered within models with universal, hence real, gaugino masses. It was shown that the phases have no significant effect on the LSP relic abundance but, however, they have a substantial impact on the LSP detection rates. 
In this paper we study the cosmological implications of the gaugino phases. In particular, we consider the D-brane model, recently proposed in Ref. [8], which allows large value of SUSY phases without exceeding the experimental upper limit on the the neutron and electron EDMs Ref. [4, []. It turns out that the LSP of this model, depending on the ratio between $M_{1}$ and $M_{2}$, could be bino or wino like. In the region where the EDMs satisfy the upper (1), the mass of the LSP is very close to the lightest chargino. Hence, in this case, the co-annihilation between the LSP and the lightest chargino becomes very important and it greatly reduces the relic density. The phases have no important effect on the LSP relic abundance as in the case studied in Ref. [7]. However, their effect on the detection rates is very significant and it is larger than what is found in the case of real gaugino masses [7].

In section 2 we briefly review the formula of the soft SUSY breaking terms in $D$-brane model [8]. We also study the effect of the phases on the LSP mass and its composition. In section 3 we compute the relic density of the LSP including the co-annihilation with the lightest chargino. We also comment on the effect of SUSY phases on the LSP detection rate. We give our conclusions in section 4.

\section{Non-universal gaugino masses}

In the framework of the MSSM and the minimal supergravity (SUGRA) the universality of gaugino masses is usually assumed, i.e., $M_{a}=M_{1 / 2}$ for $a=1,2,3$. Despite the simplicity of this assumption, it is a very particular case and there exist classes of model in which non-universal gaugino masses can be derived [9].

A type I string derived model, which has recently proposed in Ref. [8] leads to nonuniversal gaugino masses. This property, as emphazised in Ref. [4, 5], is very important for the cancellation mechanism, mentioned in the previous section. It has been shown that for this model there exists a special region in the parameter space where both the electron and the neutron EDMs satisfy the experimental constraint (1) and large values of SUSY phases and light SUSY spectrum are allowed.

The soft SUSY breaking terms in type I string theories depend on the embedding of the standard model (SM) gauge group in the D-brane sector. In case of the SM gauge group is not associated with a single set of branes the gaugino masses are non-universal [8]. 
If the $S U(3)_{C}$ and $U(1)_{Y}$ are associated with one set of five branes (say $5_{1}$ ) and $S U(2)_{L}$ is associated with a second set $5_{2}$. The soft SUSY breaking terms take the following form [8]

$$
\begin{aligned}
& M_{1}=\sqrt{3} m_{3 / 2} \cos \theta \Theta_{1} e^{-i \alpha_{1}}=M_{3}=-A, \\
& M_{2}=\sqrt{3} m_{3 / 2} \cos \theta \Theta_{2} e^{-i \alpha_{2}},
\end{aligned}
$$

where $A$ is the trilinear coupling. The soft scalar masses squared are given by

$$
\begin{aligned}
& m_{Q}^{2}=m_{L}^{2}=m_{H_{1}}^{2}=m_{H_{2}}^{2}=m_{3 / 2}\left(1-3 / 2 \sin ^{2} \theta\right) \\
& m_{D}^{2}=m_{U}^{2}=m_{E}^{2}=m_{3 / 2}\left(1-3 \cos ^{2} \theta\right)
\end{aligned}
$$

and $\Theta_{1}^{2}+\Theta_{2}^{2}=1$. In this case, by using the appropriate field redefinitions and the $R$ rotation we end up with four physical phases, which can not be rotated away. These phases can be chosen to be: the phase of $M_{1}\left(\phi_{1}\right)$, the phase of $M_{3}\left(\phi_{3}\right)$, the phase of $A$ $\left(\phi_{A}\right)$, and the phase of $\mu\left(\phi_{\mu}\right)$. The phase of $B$ is fixed by the condition that $B \mu$ is real. We notice that at the GUT scale $\phi_{1}=\phi_{3}=\phi_{A}=\alpha_{1}-\alpha_{2}$ while the phase of $\mu$ is arbitrary and scale independent.

The effect of these phases on the EDM of the electron and the neutron (by taking into account the cancellation mechanism between the different contributions), has been examined in Ref. [4, 5]. It was shown that large values of these phases can be accommodated and the electron and neutron EDMs satisfy the experimental constraint. It is interesting to note, however, that the EDMs impose a constraint on the ratio $M_{1} / M_{2}$. In fact, in order to have an overlap between the electron and neutron EDM allowed regions, $M_{2}$ should be smaller than $M_{1}$. In particular, as explained in Ref. [4], a precise overlap between these two regions occurs at $\Theta_{1}=0.85$. Such constraint has an important impact on the LSP. In this case we have the following ratios of the gaugino masses at the string scale

$$
\left|M_{3}\right|:\left|M_{2}\right|:\left|M_{1}\right|=1: \frac{\Theta_{2}}{\Theta_{1}}: 1
$$

where $\frac{\Theta_{2}}{\Theta_{1}}<1$. So that $M_{2}$ is the lightest gaugino at GUT scale. However, at the weak scale we approximately have

$$
\left|M_{3}\right|:\left|M_{2}\right|:\left|M_{1}\right|=7: 2 \frac{\Theta_{2}}{\Theta_{1}}: 1,
$$

since $\alpha_{1}: \alpha_{2}: \alpha_{3} \simeq 1: 2: 7$ at $M_{Z}$. In Figure (1) we show the running values for $\left|M_{i}\right|$ with $m_{3 / 2}$ of order $100 \mathrm{GeV}$ and $\Theta_{1}=0.85$. In our analysis we restrict ourselves to the 
region found in Ref. [4], where the electron and neutron EDMs are smaller than the limit (1), i.e., we take $\tan \beta \simeq 2, \theta=0.2, \Theta_{1}=0.85, \phi_{\mu} \simeq 10^{-1}$ and $\phi_{1} \simeq(1-1.5 \pi)$.

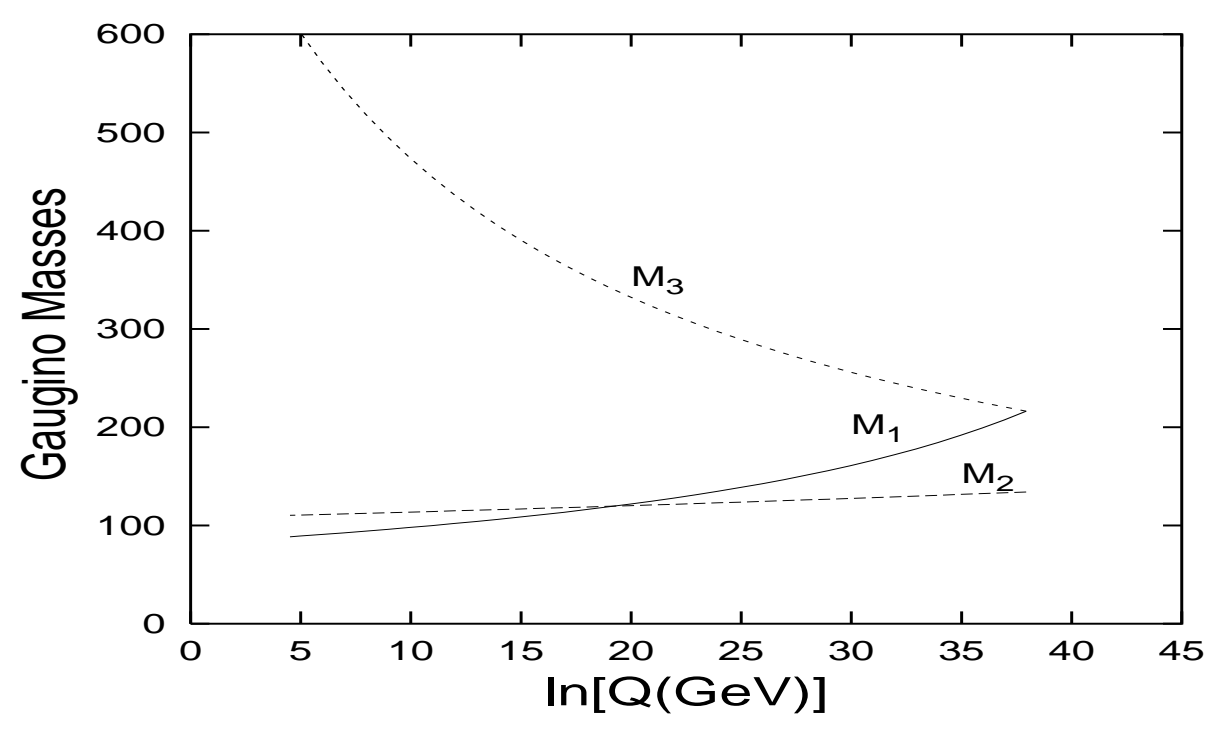

Figure 1: The running values for $\left|M_{i}\right|$ from GUT scale to the weak scale

This restriction suggests that in this scenario the lightest neutralino is a bino like. Indeed we find that the lightest neutralino, which in general is a linear combination of the Higgsinos $\tilde{H}_{1}^{0}, \tilde{H}_{2}^{0}$ and the two neutral gaugino $\tilde{B}^{0}$ (bino) and $\tilde{W}_{3}^{0}$ (wino)

$$
\chi=N_{11} \tilde{B}+N_{12} \tilde{W}^{3}+N_{13} \tilde{H}_{1}^{0}+N_{14} \tilde{H}_{2}^{0}
$$

is bino like with the gauge function $f_{g}=\left|N_{11}\right|^{2}+\left|N_{12}\right|^{2} \simeq 0.98$. Moreover it turns out that the LSP mass is close to the lightest chargino mass which is equal to the mass of the next lightest neutralino $\left(\tilde{\chi}_{2}^{0}\right)$. Figure $(2)$ shows that the mass splitting between LSP and the lightest chargino $\Delta m_{\chi^{+}}=m_{\chi_{1}}^{+} / m_{\chi}-1$ is less than $20 \%$. Therefore the co-annihilations between the bino and the chargino, as well as the next to lightest neutralino, are very important and have to be included in the calculation of the relic density.

\section{Relic Abundance and Co-annihilation effect}

In this section we compute the relic density of the LSP. Moreover, we study the effect of the SUSY CP violating phases and the co-annihilation on both the relic density and the upper bound of the LSP mass. As usual, since the LSP is bino like, the annihilation 


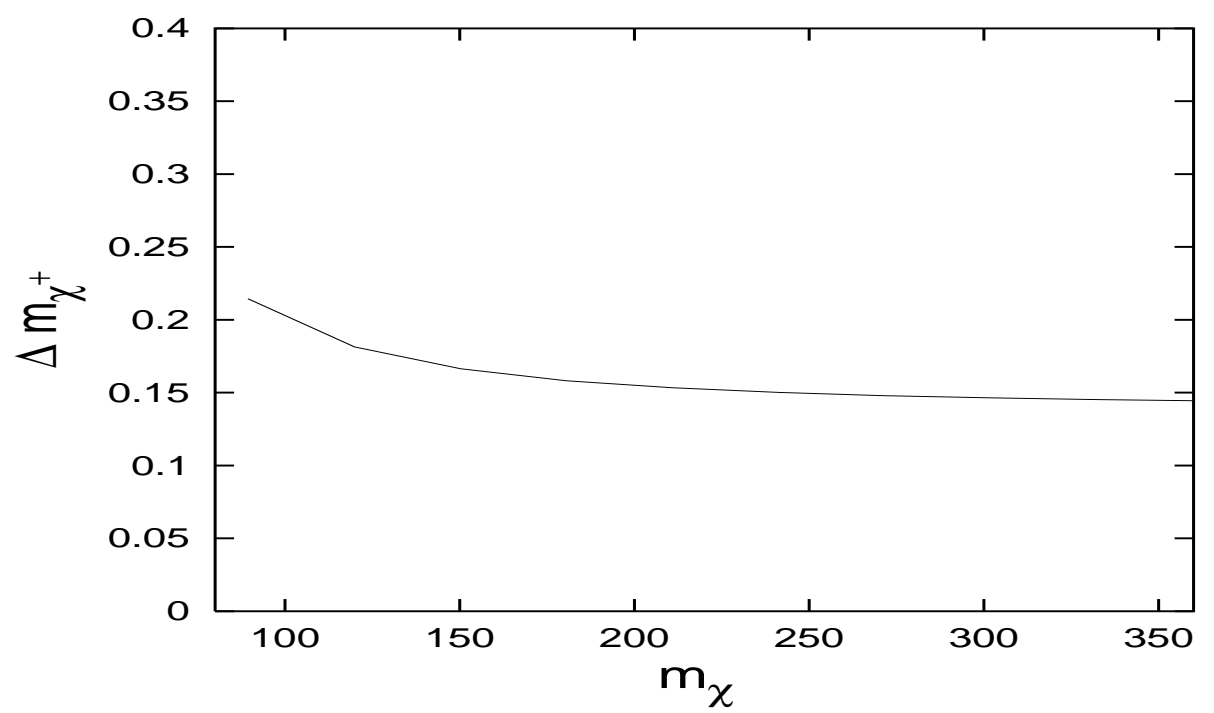

Figure 2: The mass splitting $\Delta m_{\chi^{+}}=m_{\chi_{1}}^{+} / m_{\chi}-1$ versus the LSP mass.

is predominantly into leptons by the exchange of the right slepton. Without the coannihilation, the constraint on the relic density $0.025<\Omega_{L S P} h^{2}<0.22$ imposes sever constraint on the LSP mass, namely $m_{\chi}<150 \mathrm{GeV}$. As shown in Figure (3), the SUSY phases have no any significant effect in relaxing such sever constraint.

We now turn to the calculation of the cosmological relic density of the LSP by including the co-annihilation of $\chi$ with $\chi_{1}^{+}$and $\tilde{\chi}_{2}^{0}$. As shown in Figure (3) the $\Omega_{L S P} h^{2}$ increases to unacceptable high values as $m_{\chi}$ approaches $300 \mathrm{GeV}$. This results imposes severe constraints on the entire parameter space. Therefore, in order to reduce the LSP relic density to an acceptable level, it is very important to include the co-annihilation. Several studies, which explain the effect of the co-annihilation with the next to lightest SUSY particle (NLSP), have been recently reported [10]. In these studies it was shown that, in the models with large $\tan \beta$, the NLSP turns out to be stau and its co-annihilation with the LSP is crucial to reduce the relic density to an acceptable region.

By following Ref. [11], we define the effective number of the LSP degrees of freedom

$$
g_{e f f}=\sum_{i} g_{i}\left(1+\Delta_{i}\right)^{3 / 2} e^{-\Delta_{i} x}
$$

where $\Delta_{i}=m_{i} / m_{\tilde{\chi}_{1}^{0}}-1, x=m_{\tilde{\chi}_{1}^{0}} / T$ with $T$ is the photon temperature and $g_{i}=2,4,2(i=$ $\left.\tilde{\chi}_{1}^{0}, \tilde{\chi}_{1}^{+}, \tilde{\chi}_{2}^{0}\right)$ is the number degrees of freedom of the particles. Note that the neutralinos $\chi_{1,2}^{0}$ and chargino $\chi^{ \pm}$, which are Majorana and Dirac fermions, have two and four degrees 


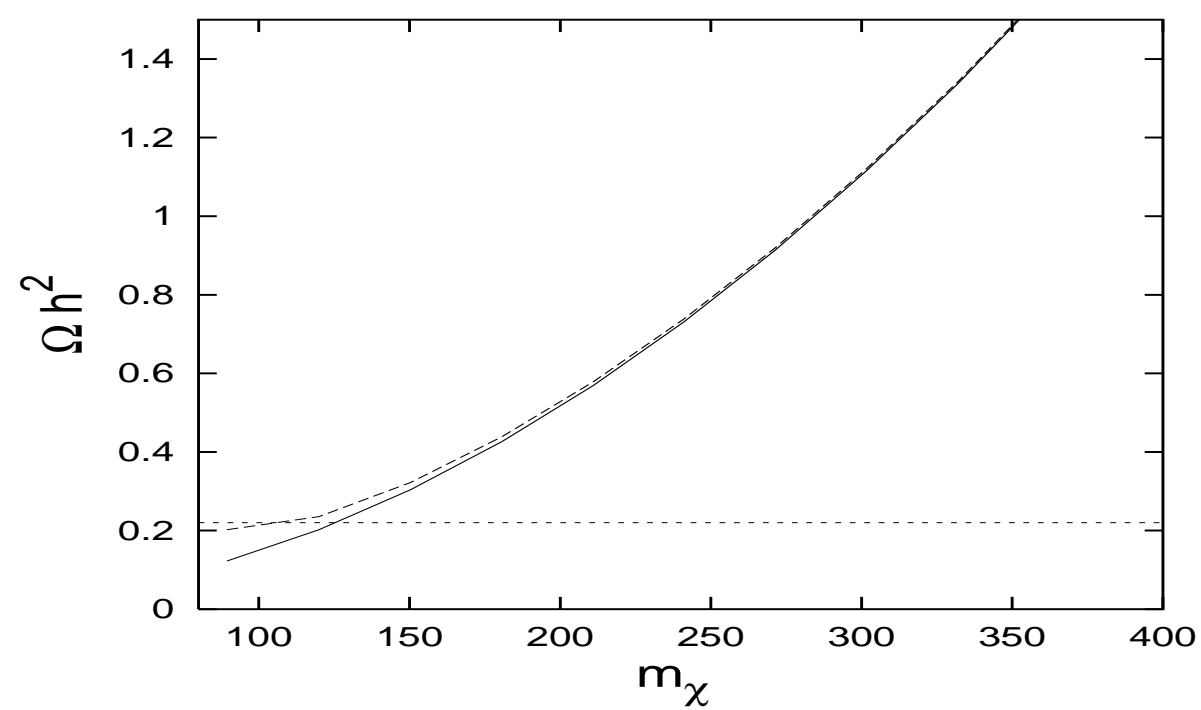

Figure 3: The LSP relic abundance without co-annihilation versus is mass, solid line corresponds to non vanishing phases while the dashed lines correspond to vanishing phases.

of freedom respectively. The Boltzmann equation for the total number density $n=\sum_{i} n_{i}$ is given by

$$
\frac{d n}{d t}=-3 H n-\left\langle\sigma_{e f f} v\right\rangle\left(n^{2}-\left(n^{e q}\right)^{2}\right),
$$

where $H$ is the Hubble parameter, $v$ is the relative velocity of the annihilation particles. The number density in the thermal equilibrium $n^{e q}$ is given by $n_{i} / n \simeq n_{i}^{e q} / n^{e q}=r_{i}$. The effective cross section, $\sigma_{e f f}$ is defined by

$$
\sigma_{e f f}=\sum_{i, j} \sigma_{i j} r_{i} r_{j}
$$

and $\sigma_{i j}$ is the pair annihilation cross section of the particle $\chi_{i}$ and $\chi_{j}$. Here $r_{i}$ is given by

$$
r_{i}=\frac{g_{i}\left(1+\Delta_{i}\right)^{3 / 2} e^{-\Delta_{i} x}}{g_{e f f}}
$$

Due to the fact that the LSP is almost pure bino, the co-annihilation processes go predominantly into fermions. However, since the coupling of $\tilde{\chi}_{2}^{0}-f-\tilde{f}$ is proportional to $Z_{2 j}$, this coupling is smaller than the corresponding one of $\tilde{\chi}_{1}^{+}-f-\tilde{f}^{\prime}$. We found that the dominant contribution is due to the co-annihilation channel $\tilde{\chi}_{1}^{+} \chi \rightarrow f \bar{f}$. We also include the $\tilde{\chi}_{1}^{+} \chi \rightarrow W^{+} \gamma$ channel which is estimated to give a few percent contribution. Then, we can calculate the relic abundance from the equation

$$
\Omega_{\chi} h^{2} \simeq \frac{1.07 \times 10^{9} \mathrm{GeV}^{-1}}{g_{*}^{1 / 2} M_{P} x_{F}^{-1} \int_{x_{f}}^{\infty}\left\langle\sigma_{e f f} v\right\rangle x^{-2} d x} .
$$


Here $M_{P}$ is the Planck scale, $g_{*} \simeq 81$ is the effective number of massless degrees of freedom at freeze out and $x_{F}$ is given by

$$
x_{F}=\ln \frac{0.038 g_{e f f} M_{P}(c+2) c m_{\tilde{\chi}_{1}^{0}}\left\langle\sigma_{e f f} v\right\rangle\left(x_{F}\right)}{g_{*}^{1 / 2} x_{F}^{1 / 2}},
$$

the constant $c$ is equal to $1 / 2$. In Figure(4) we plot the values of the LSP relic abundance $\Omega_{\chi} h^{2}$ values versus $m_{\chi}$. These values have been estimated using eq.(10) with including the co-annihilations.

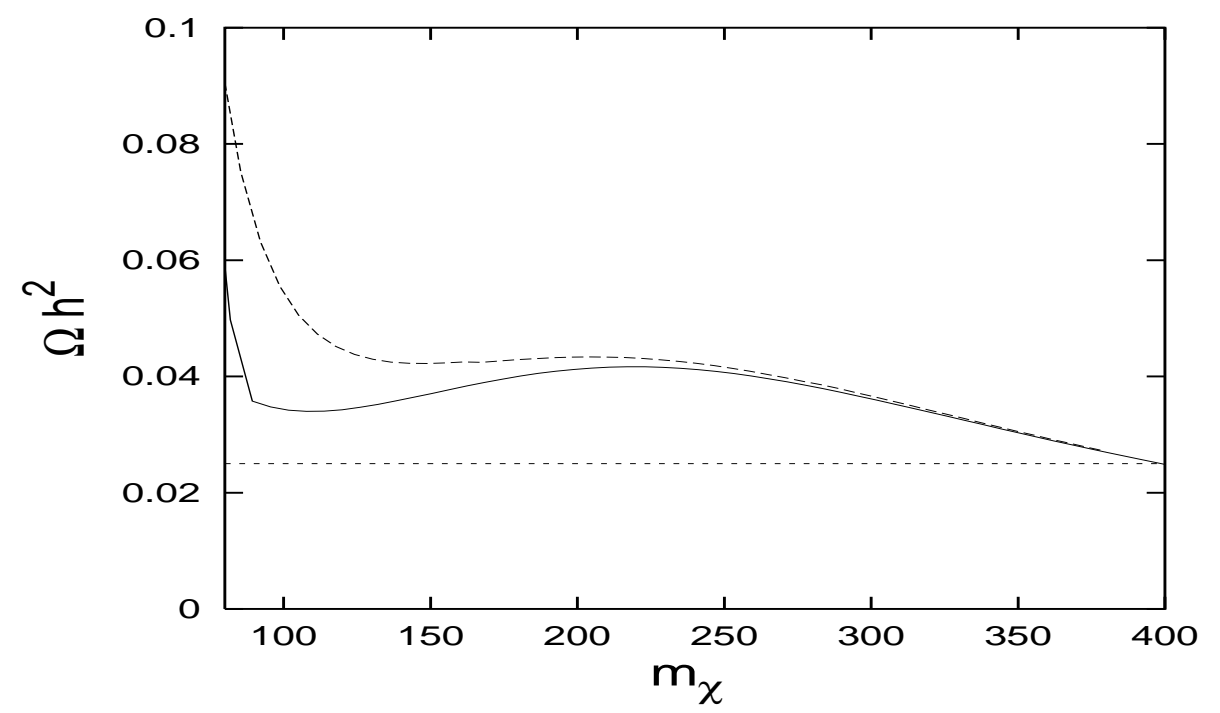

Figure 4: The LSP relic abundance with co-annihilation versus its mass, solid line corresponds to non vanishing phases while the dashed lines correspond to vanishing phases.

The results in Figure (4) show how the co-annihilation processes can play a crucial rule in reducing the values of $\Omega_{\chi} h^{2}$. By means of the lower bound on the relic density $\Omega_{\chi} h^{2}>0.025$ leads to $m_{\chi}<400 \mathrm{GeV}$. Also, here the effect of the SUSY phases is insignificant and the same upper bound of the LSP mass is obtained for vanishing and non vanishing phases.

It is worth mentioning that the gaugino phases, especially the phase of $M_{3}$, have a relevant impact on generating large $\phi_{A}$ at the electroweak $(\mathrm{EW})$ scale. It dominantly contributes to the phase of the $A$-term during the renormalization from the GUT scale to EW scale. Thus the radiative corrections to $\phi_{A}$ are very small and the phase of $A$ can be kept large at EW. However, as we were shown, such large phases are not effecting for the LSP mass and the relic abundance. In fact, this result can be explained as follow: 
the LSP is bino like (so it slightly depends on the phase of $\mu$ ) and the contribution of the phases can be relevant if there is a significant mixing in the sfermion mass matrix. In the class of models we consider the off diagonal element are much smaller than the diagonal element.

As shown in Ref. [6], the SUSY phases have a significant effect on the direct detection rate $(R)$ and indirect detection rate $(\Gamma)$ : the phase of $\phi_{A}$ increases the values of $R$ and $\Gamma$ - Furthermore the enhancement of the ratios of the rates with non vanishing $\phi_{A}$ to the rates in the absence of this phase are even larger than what is found in Ref. [6]. Indeed due to the gluino contribution (through the renormalization) the phase $\phi_{A}$ can get larger values at EW scale.

\section{Conclusions}

We considered type I string derived model which leads to non-universal gaugino masses and phases. As recently shown, these non-universality is very important to have sufficient cancellations among different contributions to the EDM. Moreover the EDM of the electron and neutron imposed constraint on the ratio of the gaugino masses $M_{1}$ and $M_{2}$. This implies that the mass of the LSP (bino-like) is close to the lightest chargino mass. The co-annihilation between the LSP and lightest chargino is crucial to reduce the relic density to an interesting region. The phases have no significant effect on the LSP mass and its relic density, but have a substantial effect on the direct and indirect detection rates.

\section{Acknowledgments}

This work is supported by the Spanish Ministerio de Educacion y Cultura research grant. 


\section{References}

[1] M. Dugan, B. Grinstein, and L. Hall, Nucl. Phys. B 255 (1985) 413.

[2] T. Ibrahim and P. Nath, Phys. Lett. B 418 (1998) 98;

Phys. Rev. D D57 (1998) 478; Erratum idid D58 (1998) 019901.

[3] S. Barr and S. Khalil Phys. Rev. D 61 (2000) 035005.

[4] M. Brhlik, L. Everett, G. Kane, and J. Lykken, Phys. Rev. Lett. 83 (1999) 2124 ;

M. Brhlik, L. Everett, G. Kane, and J. Lykken, hep-ph/9908326.

[5] E. Accomando, R. Arnowitt, and B. Dutta, hep-ph/9909333.

[6] S. Khalil and Q. Shafi, Nucl. Phys. B 564 (1999) 19.

[7] U. Chattopadhyay, T. Ibrahim, and P. Nath, Phys. Rev. D 60 (1999) 063505;

T. Falk, A. Frestl, and K. Olive, Phys. Rev. D 59 (1999) 055009 ;

T. Falk and K. Olive, Phys. Lett. B 375 (1996) 196;

T. Falk and K. Olive, Phys. Lett. B 354 (1995) 99 .

[8] L. Ibanez, C. Munoz, and S. Rigolin, Nucl. Phys. B 553 (1999) 43.

[9] J. Feng, T. Moroi, and L. Randall, Phys. Rev. Lett. 83 (1999) 1731 ;

K. Huitu, Y. Kawamura, T. Kobayashi, and K. Puolamaki, hep-ph/9903528;

G. Anderson, H. Baer, C. Chen, and X. Tata, hep-ph/9903370 ;

C. Chen, M. Drees, and J. Gunion, Phys. Rev. D 55 (1997) 347, Erratum idid D60 (1999) 039901.

[10] A. Lahanas, D. Nanopoulos, and V. Spanos, hep-ph/9909497;

M. Gomez, G. Lazarides, and C. Pallis, hep-ph/9907261 ;

J. Ellis, T. Falk, K. Olive, and M. Srednicki, hep-ph/9905481.

[11] K. Griest and D. Seckel, Phys. Rev. D 43 (1991) 3191.

[12] G. Jungman, M. Kamionkowski, and K. Griest, Phys. Rep. 267 (1996) 195. 